\title{
Widening the 2D/3D Perovskite Family for Efficient and Thermal Resistant Solar Cells by the Use of Secondary Ammonium Cations
}

Jesús Rodríguez-Romero, ${ }^{\mathrm{a},+}, *$ Jesús Sanchez-Diaz, ${ }^{\mathrm{a}, \mathrm{b},+}$ Carlos Echeverría-Arrondo, ${ }^{\mathrm{a}}$ Sofia Masi, ${ }^{a}$ Diego Esparza, ${ }^{\mathrm{b}}$ Eva M. Barea, ${ }^{\mathrm{a}}$ and Iván Mora-Seró. ${ }^{\mathrm{a}, *}$

\author{
a Institute of Advanced Materials (INAM), Universitat Jaume I, 12006 Castelló, Spain \\ b Unidad Académica de Ingeniería Eléctrica, Universidad Autónoma de Zacatecas, \\ México
}

+ These authors contributed equally

Email: rodrigje@uji.es, sero@uji.es

\begin{abstract}
While 2D/3D layered perovskites have been the object of comprehensive researches principally focused on increasing the long-term stability observed in 3D perovskites, significant opportunities are still open concerning the application of different kinds of cations which are outside the sphere of primary amines, which are the cations most usually applied. Our results demonstrate that the materials and the solar cells prepared with dipropylammonium iodide (DipI), a bulky secondary ammonium cation of small size, lead to obtaining not only efficient and thermodynamically stable materials but also robust towards heat stress. Time-resolved studies point out longer carrier's lifetime for 2D/3D layered perovskites fabricated with this bulky cation than systems based on bulky primary ammonium cations, which allowed us to obtain PCE $=12.51 \% \quad(n=10)$, $15.78 \%(n=50)$ and $17.90 \%(n=90)$. We determine that the concentration of perovskite material after $240 \mathrm{~min}$ at $100^{\circ} \mathrm{C}$ is until $575 \%$ greater in the $2 \mathrm{D} / 3 \mathrm{D}$ perovskite $(\mathrm{n}=10)$ than the observed in $3 \mathrm{D}$ perovskite films. The material stability also improves the thermal stability of the photovoltaic devices presenting an efficiency drop of just $4 \%$ for $n=50$ and $n=10$ after thermal annealing while the performance drop for reference 3D samples in the same conditions was of higher than $80 \%$.
\end{abstract}


The impressive performance that photovoltaic technology based on halide perovskite materials reached during the last years is just overshadowed by their long-term stability limitations, which could limit their massive commercial implantation. ${ }^{1-4}$ Throughout, two-dimensional halide perovskites (2D HPs) have emerged as a potential alternative to overcome these issues, although this class of materials has been focused mainly on increasing the moisture-resistant properties. ${ }^{5,6} 2 \mathrm{D}$ or $2 \mathrm{D} / 3 \mathrm{D}$ HPs are prepared by the total or partial substitution of the small cations forming 3D perovskites, typically methylammonium (MA), formamidinium (FA), or Cesium, by cations with bigger size. The introduction of larger cations rift the conventional 3D arrangement of cornersharing $\mathrm{PbI}_{6}^{4-}$ octahedra, generating layered materials where, depending on the cation and the stoichiometry, it is possible to obtain different phases, for example, RuddlesdenPopper, ${ }^{7}$ Dion-Jacobson, ${ }^{8}$ and an interesting material denominated as Alternating Cations in the Interlayer space, ACI. ${ }^{9}$

Structurally, 2D, with bulky organic cations, or 2D/3D HPs, with both bulky and small organic cations, are arrangements where the inorganic layers of the corner-sharing $\mathrm{PbI}_{6}{ }^{4-}$ octahedra are sandwiched between the organic bilayers. ${ }^{10}$ The number of these inorganic layers, including the small cation on the 2D/3D case, (knows as $n$ ) can be easily tuned by different stoichiometric proportions between the small and big cations, keeping in mind the desired chemical formulation according to the chosen phase. ${ }^{9}$ The possibility to include bulky cations with not only unlimited structural diversity but also with the required physicochemical properties has led to obtain materials with impressive performance not only in photovoltaic technology, ${ }^{6,11-13}$ but also as photodetectors ${ }^{14}$ and light-emitting diodes. ${ }^{15}$

As a general rule, the bulky cations used to date come from ammonium salts derived from primary amines, as ammonium halides derived from alkyl-saturated chains, 7,16 alkenyl, ${ }^{17}$ aryl-alkyl, ${ }^{5,18,19}$ and aryl amines. ${ }^{20-22}$ The principal interest of the study of 2D/3D perovskites for photovoltaic applications has been the increase of stability to moisture due to the hydrophobic nature of the employed bulky cation. ${ }^{5}$ However, tailoring the interaction between the organic and inorganic parts is also extremely appealing; consequently, the incorporation of bulky cations, with different 
functionalization, has awakened the interest of the scientific community, with the aim to obtain materials where the specific functionalization of the bulky cations can provide novel and improved properties. Different researchers have tested conjugated chromophores, ${ }^{19}$ charge transfer complexes, ${ }^{23}$ polymers, ${ }^{24}$ and even cations with two ammonium heads (these particular examples are represented by di-amine cations with secondary-primary and quaternary-primary ammonium salts), ${ }^{25,26}$ in order to obtain specific functionalization. However, these described samples are always ammonium salts derived from primary amines.

Intuitively, the absence of secondary-, tertiary- and quaternary ammonium salts that perform the role of bulky cations in 2D HPs, could be related to a potential detrimental effect in the stability as a consequence of the decrease of the number of hydrogen bonds, which is one of the principal forces responsible for holding the perovskite structure together. However, the introduction of a secondary ammonium cation instead the primary analog introduces the possibility of a fine-tuning of the properties through molecular engineering, as it allows to include a double functionalization instead of the single one provided by primary ammonium cations, see Scheme 1. Beyond, the introduction of tertiary ammonium cations to obtain a perovskite structure, theoretically, it is possible and, with the introduction of an additional substituent, the chemistry of the cations could be huge enriched. However, on the basis of the next considerations, we hypothesize that that class of material would have poor stability. First, the number of hydrogen bonds will be lower, 2 and 1 lower than primary and secondary ammonium cations, respectively. Second, the potential observation of steric hindrance as a consequence of the three alkyl/aryl substituents located on nitrogen, it will weaken the effective interaction between the unique hydrogen and the halides, see Scheme 1. In order to explore both, the potentiality of secondary ammonium cations for the development of perovskite solar cells (PSCs) and a potential stability owing to a higher molecular weight (avoiding sublimation) and a greater number of Van der Waals interactions (to compensate for the least amount of hydrogen bonds comparing with primary ammonium salts analog), we have systematically analyzed the application of dipropylammonium iodide (DipI) as bulky cation in 2D/3D perovskite materials and its application in PSCs. The set of studied materials are represented by the general formula $\operatorname{Dip}_{2} \mathrm{MA}_{\mathrm{n}-1} \mathrm{~Pb}_{\mathrm{n}} \mathrm{I}_{3 \mathrm{n}+1}$, where Dip $=\left(\mathrm{CH}_{3} \mathrm{CH}_{2} \mathrm{CH}_{2}\right)_{2} \mathrm{NH}_{2}{ }^{+}, \mathrm{MA}=\mathrm{CH}_{3} \mathrm{NH}_{3}{ }^{+}$. We prepared materials with nominal $n=3,5,10,50$ and 90 . Through optical studies, we compare with 
result reported in the literature and demonstrate that the application of smaller cations $\left(\left(\mathrm{CH}_{3} \mathrm{CH}_{2} \mathrm{CH}_{2}\right)_{2} \mathrm{NH}_{2}^{+}\right)$leads to larger carrier's lifetime than cations with the same number of $\mathrm{CH}_{\mathrm{n}}$ unities but of longer length $\left(\left(\mathrm{CH}_{3} \mathrm{CH}_{2} \mathrm{CH}_{2} \mathrm{CH}_{2} \mathrm{CH}_{2} \mathrm{CH}_{2}\right) \mathrm{NH}_{3}{ }^{+}\right){ }^{27} \mathrm{We}$ fabricated PSCs with the highest $\mathrm{n}$ materials obtaining a significant performance and thermal stability, increasing as a function of $n$, demonstrating the suitability of secondary ammonium cation for the development of PSCs.

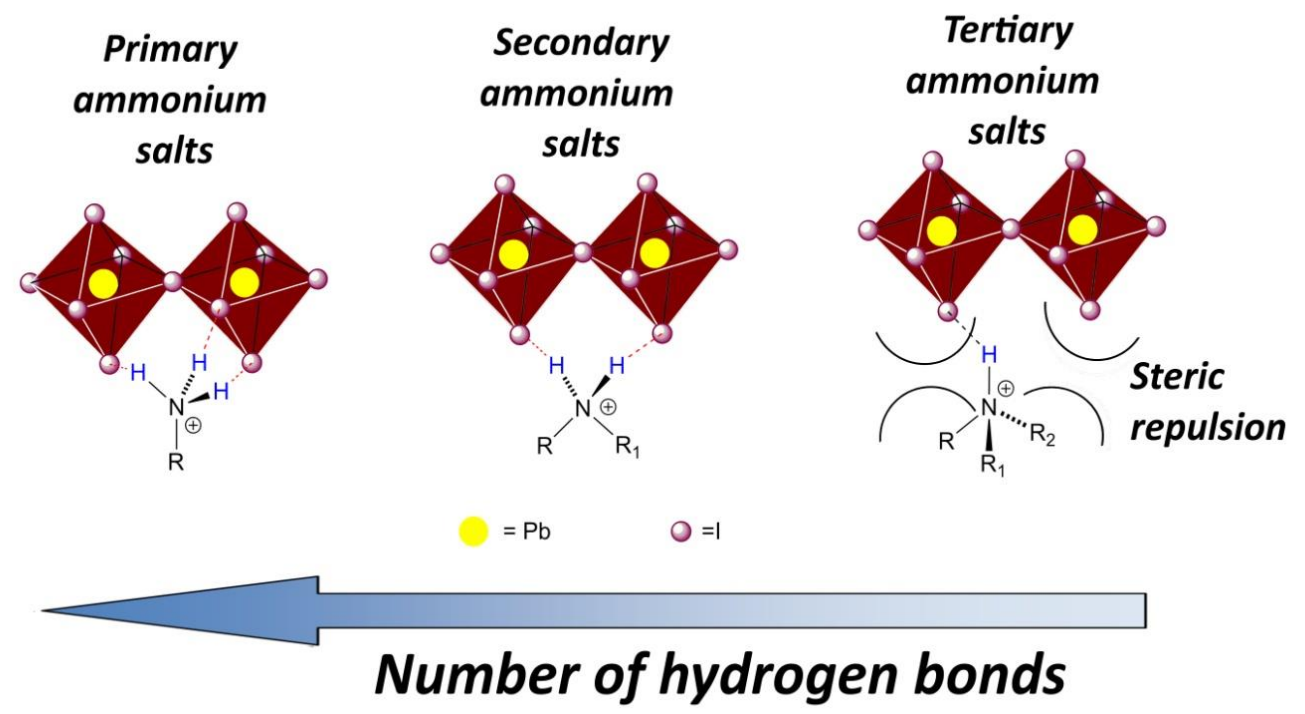

Scheme 1. Comparison of primary, secondary and tertiary ammonium cations as a function of the number of potential hydrogen bonds. The presence of two substituents, $\mathrm{R}$ and $\mathrm{R}_{1}$, in the secondary ammonium derivative opens the possibility to include multifunctional cations within the structure in contrast to primary ammonium salt, where the functionalization is restricted to just one kind of substituent. Despite the introduction of a tertiary ammonium cation is theoretically possible, the lower number of hydrogen bonds between the unique hydrogen on the molecule with the halide of the perovskite, together the potential observation of steric hindrance, would limit its study.

To determine the formation of $\operatorname{Dip}_{2} \mathrm{MA}_{\mathrm{n}-1} \mathrm{~Pb}_{\mathrm{n}} \mathrm{I}_{3 \mathrm{n}+1}$ layered perovskites, we prepare a set of films with nominal $n=3,5,10,50$ and 90 . The films were prepared through the dripping of the perovskite precursor solution over a $\mathrm{SnO}_{2}$-compact layer, using ethyl acetate as antisolvent. XRD characterization, see Figure 1a, demonstrate that the material with $n=3$ exhibits a complex design with diffraction peaks between 3.58 and $10.66^{\circ}$, which are the evidence of the $2 \mathrm{D}$ nature of this material. ${ }^{7}$ Similar complex patterns have been previously reported when were used bulky cations, like the derived of 5 -aminovaleric acid, in the assembling of $2 \mathrm{D} / 3 \mathrm{D}$ perovskites. ${ }^{11}$ Furthermore, this 
XRD presents a broad peak at $14.16^{\circ}$, which has been previously assigned to horizontal (111) preferential growth in 2D perovskites but also the (110) plane in 3D perovskites. ${ }^{7}$ These results lead us point to the presence of $2 \mathrm{D} / 3 \mathrm{D}$ domains with different $\mathrm{n}$ but also a $3 \mathrm{D}$ perovskite phases within our material, as it is conventionally reported for $2 \mathrm{D} / 3 \mathrm{D}$ perovskites with primary ammonium cation. ${ }^{6}$ For films with $n=5$ and 10 , it is possible to see diffraction peaks below $10^{\circ}$, see Figure 1a, which implies a considerable amount of the $2 \mathrm{D} / 3 \mathrm{D}$ component in the material. In contrast, for the perovskite with $\mathrm{n}=50$ and 90 , the XRD data displays just the peaks associated with the $3 \mathrm{D}$ perovskite, although the absence of diffraction peaks related to $2 \mathrm{D} / 3 \mathrm{D}$ phase does not involve mandatorily that this material is not present in the film, but its concentration is below the limit of detection, as has been demonstrated in cations like guanidinium. ${ }^{28}$ On the other hand, the analysis of the full-width at the half-maximum (FWHM) for the dominant peak at $14.16^{\circ}$, see Figure $1 \mathrm{~b}$, shows a progressive decrease, especially abrupt for high $\mathrm{n}$, indicating an increase of the crystallite size as the 3D phase becomes dominant.

(a)

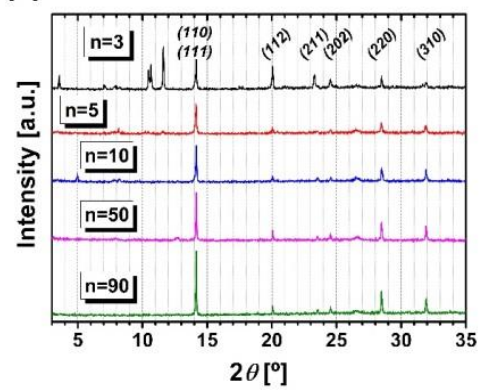

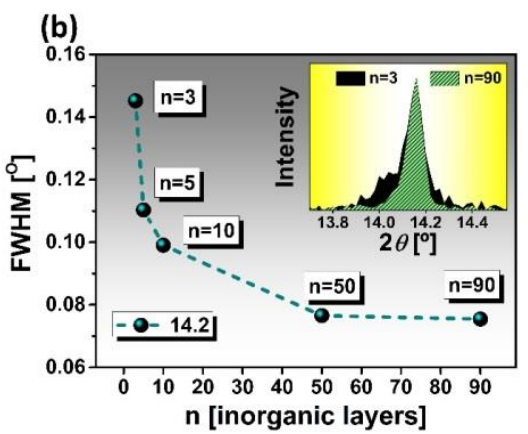

Figure 1. Morphological studies by XRD. (a) XRD for $\operatorname{Dip}_{2} M_{A} A_{n-1} P b_{n} I_{3 n+1}$ with $n=3,5$, 10, 50 and 90. (b) FWHM for the peak at $14.16^{\circ}$.

$\operatorname{Dip}_{2} \mathrm{MA}_{\mathrm{n}-1} \mathrm{~Pb}_{\mathrm{n}} \mathrm{I}_{3 \mathrm{n}+1}$ layered perovskites present similar optical properties in terms of absorbance spectra see Figure 2a, and the steady-state photoluminescence (PL), see Figure $2 b$. The complete set of studied samples exhibit similar spectral profiles. Notably, under the employed conditions, we could not observe the characteristic excitonic transitions observed in layered materials, where the stability of the excitons allow to observe them at room temperatures. ${ }^{29}$ The bandgap, calculated from the Tauc plot, determined for the samples with $\mathrm{n}=3$ and 5 was $1.57 \mathrm{eV}$, which is slightly redshifted comparing with the calculated for materials with $n=10(1.60 \mathrm{eV})$. Besides, it was possible to observe the presence of an Urbach tail, which agrees with crystal disorder and the presence of multiple phases observed in the XRD results. The films prepared 
with $n=10$ exhibit a slight weaker absorbance near the IR edge, while in the UV region this reduction becomes noteworthy. Comparable behavior was observed for the film with $n=50$, although the change is less pronounced. Finally, the film with $n=90$ does not show any significant difference with the 3D perovskite. These results confirm the expected correlation between $\mathrm{n}$ and the absorbance. As $\mathrm{n}$ decreases, lower absorbance (predominantly in the region of 400 to $550 \mathrm{~nm}$ ). This result has special significance in the behavior of light-absorbers materials, considering the direct relationship of the absorbance with the short circuit current $\left(J_{s c}\right)$ and therefore the PCE. On the other hand, we can determine that the PL emission is essentially at the same wavelength for all the evaluated materials, see Figure $2 b$, indicating the emission from the $3 \mathrm{D}$ domains, ${ }^{29,30}$ regardless of the higher concentration of dipropylammonium iodide in the materials with the higher $2 \mathrm{D} / 3 \mathrm{D}$ character (samples with $\mathrm{n}=3$ and 5 ) pointing to a funneling effect from low $\mathrm{n}$ domains with wider band gap to the 3D domains with the narrowest bandgap.
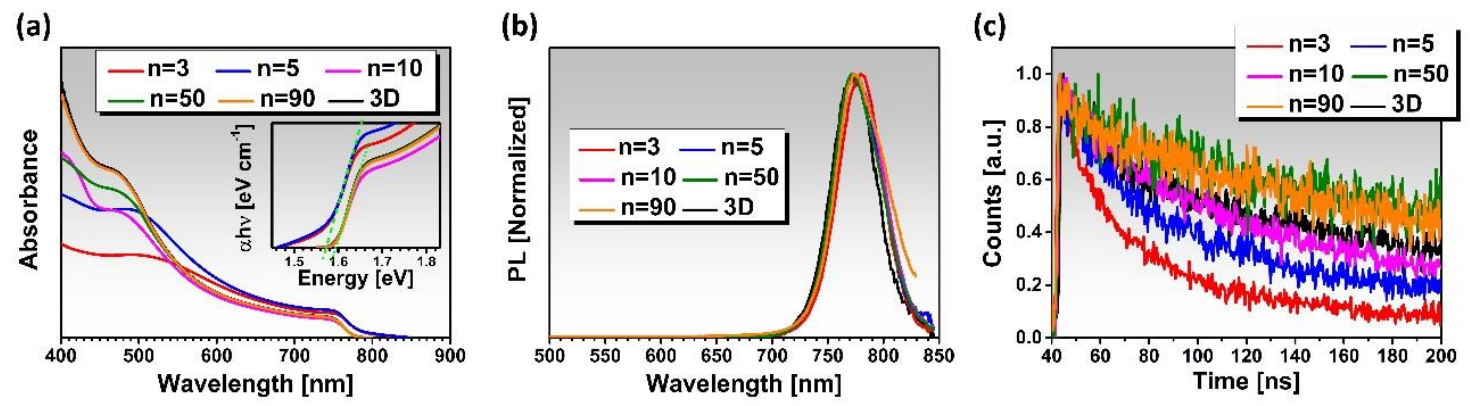

Figure 2. Optical characterization. (a) Absorbance and (b) PL measurements of $\mathrm{Dip}_{2} \mathrm{MA}_{\mathrm{n}-1} \mathrm{~Pb}_{\mathrm{n}} \mathrm{I}_{3 \mathrm{n}+1}$ with $\mathrm{n}=3,5,10,50,90$ and 3D. (c) Time Correlated Single Photon Counting measurements for $\operatorname{Dip}_{2} \mathrm{MA}_{\mathrm{n}-1} \mathrm{~Pb}_{\mathrm{n}} \mathrm{I}_{3 \mathrm{n}+1}$ with $\mathrm{n}=3,5,10,50,90$ and 3D. The traces and fitting results are available in the Figure S3.

The remarkable absence of the excitonic peaks in UV-Vis and PL spectroscopy, like those perceived in layered perovskites, ${ }^{5,7}$ has been earlier reported for materials where the perovskites were fabricated using different proportions of cations as methylammonium (MA) and guanidinium (GA). ${ }^{28,31}$ It is important to note that guanidinium displays a slightly larger size than those required to obtain a perfect fit in the hollows and provide an ideal perovskite structure. Vega et $\mathrm{al}^{28}$ reported the optical study of materials with different ratios of MA/GA. They found that at temperatures below $70 \mathrm{~K}$, it was possible to observe only the characteristic emission associated with 3D perovskites (near $800 \mathrm{~nm}$ ), even for the formulation with MA:GA ratios of 50:50; 
while the expected excitonic emission observed at 500-600 nm, it was possible to detect it only at temperatures as low as $20 \mathrm{~K}$. This observation strongly suggests that the electron-hole pair is not linked, and charges are predominantly in form of free carriers. ${ }^{29}$ This finding is also supported by the observation of De Marco et al, ${ }^{31}$ where they reported similar emission wavelengths at room temperature, independently of the concentration of guanidinium in the corresponding materials.

In spite of the similar XRD, absorbance spectra and the steady-state PL, timeresolved photoluminescence TRPL present significant charge carrier dynamics differences depending of the nominal $\mathrm{n}$, see Figure 2c. TRPL characterization was performed on $\operatorname{Dip}_{2} \mathrm{MA}_{\mathrm{n}-1} \mathrm{~Pb}_{\mathrm{n}} \mathrm{I}_{3 \mathrm{n}+1}$ films deposited on FTO-coated glass, following the method described in the experimental section. The curves were accurately fitted following a bi-exponential method and the corresponding parameters can be found in Table 1. There is clear trend that both TRPL time constants first increase and then decrease with increasing $n$ value, see Table 1 . This trend points to a clear transition from $2 \mathrm{D} / 3 \mathrm{D}$ to $3 \mathrm{D}$ character. The samples with $\mathrm{n}$ equal or lower than 50 , with a dominant $2 \mathrm{D} / 3 \mathrm{D}$ character, show a progressive increase in the fast decay, $\tau_{1}$, usually associated to radiative recombination of excitons, ${ }^{32}$ from 18.53 ns to $96.50 \mathrm{~ns}$, see Table 1 . The slow decay, $\tau_{2}$, related to trap states, ${ }^{32}$ present a similar trend and consequently, it is observed a monotonic increase in the average lifetime, $\tau_{\text {avg. }}$. The observed behavior matches accurately with the foreseen results. As "n" rises, i.e. the structure becomes mostly 3D, the effects related to quantum and dielectric confinement effects must be lower than those predicted to materials near to $2 \mathrm{D}$ limit. That fact is reflected specifically as an increase in the carrier lifetime through the limitation of the excitons' ${ }^{\prime}$ recombination. ${ }^{33}$ For example, Mao et al ${ }^{32}$ reported faster decay times, in the order of picoseconds (340 ps and $130 \mathrm{ps})$, for purely 2D materials $(\mathrm{n}=1)$; the materials that they used as bulky cation were histammonium and benzyl ammonium. In addition, Jung found that for 2D/3D perovskites with hexylamine as bulky cation, molecule with the same number of $\mathrm{CH}_{\mathrm{n}}$ unities that our cation but derived of a primary amine, the carrier's lifetime is in the order of hundreds of picoseconds for $2 \mathrm{D} / 3 \mathrm{D}$ perovskites with $\mathrm{n}=1-4,{ }^{27}$ in agreement with the observation of Mao. ${ }^{32}$ In contrast, when guanidinium was used, which is a molecule that can form interesting 2D/3D materials, ${ }^{34}$ the carrier's lifetime is in the order of hundreds of nanoseconds with a decrease in the carriers' lifetime as the concentration of guanidinium increases, ${ }^{31}$ in strong agreement with the results reported 
here. In contrast, for $n=90$ and $3 \mathrm{D}$ is observed a progressive decrease of the lifetime; in this case the fast recombination decay has been related to bimolecular recombination while the slow decay with the recombination of free carriers in the radiative channel. ${ }^{35}$

Table 1. Optical parameters for $\operatorname{Dip}_{2} \mathrm{MA}_{\mathrm{n}-1} \mathrm{~Pb}_{\mathrm{n}} \mathrm{I}_{3 \mathrm{n}+1}, \mathrm{n}=3,5,10,50$ and 90, 3D.

Detection wavelength $\lambda_{\text {det }}=773 \mathrm{~nm}$. Average lifetime is calculated as $\tau_{\text {avg }}=\sum \mathrm{A}_{\mathrm{i}} \tau_{\mathrm{i}}^{2} / \sum \mathrm{A}_{\mathrm{i}} \tau_{\mathrm{i}}{ }^{27}$

\begin{tabular}{|l|c|c|c|c|c|c|c|}
\hline & $\mathbf{E}_{\mathbf{g}}[\mathbf{e V}]$ & $\boldsymbol{\lambda}_{\mathrm{em}}[\mathbf{n m}]$ & $\boldsymbol{\tau}_{\mathbf{1}}[\mathbf{n s}]$ & $\mathbf{A}_{\mathbf{1}}[\boldsymbol{\%}]$ & $\boldsymbol{\tau}_{\mathbf{2}}[\mathbf{n s}]$ & $\mathbf{A}_{\mathbf{2}}[\mathbf{\%}]$ & $\boldsymbol{\tau}_{\mathbf{a v g}}[\mathbf{n s}]$ \\
\hline $\mathrm{n}=3$ & 1.57 & 780 & 18.53 & 30.30 & 84.15 & 69.70 & 40.6 \\
\hline $\mathrm{n}=5$ & 1.57 & 774 & 31.92 & 21.73 & 157.66 & 78.27 & 84.95 \\
\hline $\mathrm{n}=10$ & 1.60 & 774 & 59.81 & 28.44 & 345.19 & 71.56 & 146.46 \\
\hline $\mathrm{n}=50$ & 1.60 & 773 & 96.50 & 9.40 & 327.57 & 90.60 & 267.39 \\
\hline $\mathrm{n}=90$ & 1.60 & 773 & 18.77 & 1.18 & 241.92 & 98.82 & 212.15 \\
\hline $3 \mathrm{D}$ & 1.60 & 773 & 24.22 & 6.29 & 226.61 & 93.71 & 148.55 \\
\hline
\end{tabular}

In contrast with the remarks pointing that the exciton binding energies in $2 \mathrm{D} / 3 \mathrm{D}$ HPs are not affected by the size of the bulky cations, ${ }^{36}$ our findings, when we compare with studies reported by other groups using larger cations, ${ }^{28,37}$ suggest that the cation's size present an inverse relationship with the carriers lifetimes. Therefore, the use of bulky cations with an intermediate-small size could lead to obtaining materials that could exhibit longer carriers' lifetime, which has been associated with a large density of free carriers when the sample is under illumination. ${ }^{38}$

Long carrier lifetime predicts a promising behavior of this material in photovoltaic devices. Consequently, after fundamental characterization, we focused on the fabrication of planar solar cells using $\mathrm{SnO}_{2}$ and Spiro O-MeTAD as electron and hole selective contacts, respectively, and gold on top of Spiro O-MeTAD as metallic extracting contact. The fabrication of the films was following the method previously reported. ${ }^{39}$ Briefly, the substrates coated with a $\mathrm{SnO}_{2}$ compact layer were exposed during 20 min of $\mathrm{O}_{3}$. After that, the perovskite precursor solution was drop coasted and posteriorly the film was annealed at $130{ }^{\circ} \mathrm{C}$ during $10 \mathrm{~min}$.

One of the main interests of the 2D/3D HP for photovoltaics is the higher tolerance to the moisture than their 3D counterparts. The origin of improved moisture stability is in the hydrophobic character of the hydrocarbon chains. Moreover, the nature 
of the bulky cation introduces important effects in the optoelectronic and electrical properties of the resultant material as, for example, lower absorbance of the solar spectrum, ${ }^{5,7}$ higher exciton binding energies, ${ }^{36,40-42}$ higher carrier transport resistance, ${ }^{24}$ and consequently lower PCE. ${ }^{5,7}$ One of the most successful assays to bind off the negative effects related to layered materials is the fabrication of $2 \mathrm{D} / 3 \mathrm{D}$ with a $\mathrm{n}$ value near the 3D limit. This approach tries to keep the best properties of both materials: the higher stabilities towards environmental conditions provided by 2D HP and the superior photovoltaic performance observed in 3D HP., ${ }^{6,11}$ Therefore, we focused on the assembly of 2D/3D HP ( $n=10,50$ and 90) based solar cells in the planar configuration. Figure 3 shows the current density-voltage (J-V) curves for champion devices, for each of the tested materials, where it is noticeable the increase of the PCE as n rises. Short circuit currents are in similar values for $n=50$ and 90 , which validate the optical and morphological results. Together 3D perovskite, the material with $n=90$ exhibit very high fill factor (FF), which demonstrate the high efficiency in the charge collection of this material, see Table 2. However, the materials with $\mathrm{n}=50$ and 10 exhibit a systematic reduction in the fill factor, $\mathrm{FF}$, as well as open-circuit voltage, $V_{o c}$. We consider the reduction of FF is due to the higher concentration of bulky cation, considering that this behaves as an insulator, decreasing the carrier diffusion efficiency. ${ }^{43}$ Finally, the lowest PCE obtained for the material with $\mathrm{n}=10$ is strongly affected by the decrease of $J_{s c}$, influenced also by the relatively lower absorbance than the materials with $n>10$, see Figure 2a, and in which transport limitation is also influencing.

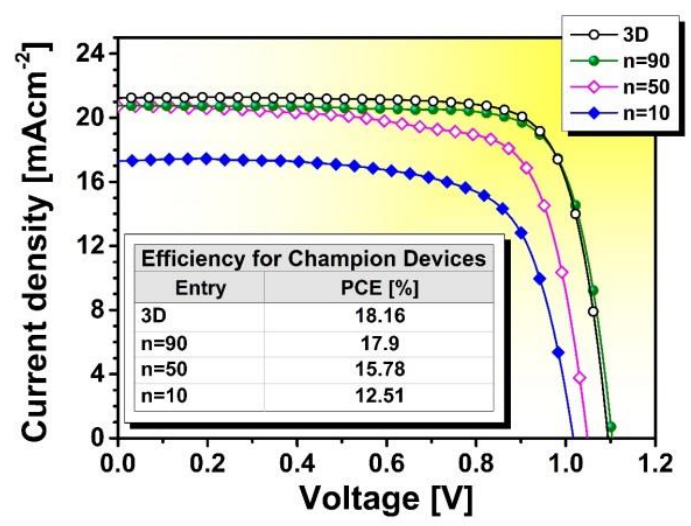


Figure 3. J-V curves for champion devices $\operatorname{Dip}_{2} \mathrm{MA}_{n-1} \mathrm{~Pb}_{n} \mathrm{I}_{3 \mathrm{n}+1}$ with $\mathrm{n}=10,50$ and 90 and $3 \mathrm{D}$ and its PCE (in table).

Table 2. Photovoltaic parameters for the champion devices obtained from the JV reverse scan. The mean values are in parenthesis. Figure S4 shows stabilized efficiency measurements and the hysteresis observed when PCE was measured in both directions.

\begin{tabular}{|l|c|c|c|c|}
\hline \multicolumn{1}{|c|}{ Sample } & $\mathbf{J}_{\mathbf{s c}}\left[\mathbf{m A c m}^{-2}\right]$ & $\mathbf{V}_{\mathbf{~ o c}}[\mathbf{m V}]$ & FF [\%] & PCE [\%] \\
\hline $3 \mathrm{D}$ & 21.25 & 1080.92 & 78.14 & 18.16 \\
& $(20.90 \pm 0.05)$ & $(1076.26 \pm 1.55)$ & $(74.62 \pm 0.39)$ & $(16.79 \pm 0.12)$ \\
\hline $\mathrm{n}=90$ & 20.77 & 1103.52 & 78.11 & 17.90 \\
& $(20.31 \pm 0.17)$ & $(1089.64 \pm 1.86)$ & $(75.50 \pm 0.48)$ & $(16.71 \pm 0.18)$ \\
\hline $\mathrm{n}=50$ & 20.73 & 1048.35 & 72.64 & 15.78 \\
& $(18.91 \pm 0.23)$ & $(1024.52 \pm 2.30)$ & $(67.72 \pm 0.87)$ & $(13.14 \pm 0.28)$ \\
\hline $\mathrm{n}=10$ & 17.45 & 1016.45 & 70.53 & 12.51 \\
& $(16.17 \pm 0.37)$ & $(965.66 \pm 9.51)$ & $(68.92 \pm 2.42)$ & $(10.73 \pm 0.39)$ \\
\hline
\end{tabular}

From the literature, it is clear to distinguish that the complete set of the salts employed as bulky cations in the manufacture of $2 \mathrm{D}, 2 \mathrm{D} / 3 \mathrm{D}$ or even $3 \mathrm{D}$ perovskites are ammonium salts derived from primary amines. ${ }^{25}$ Undoubtedly, this election should be related to the fact that the hydrogen bond is one of the most remarkable forces in the perovskite framework and, of course, one of the responsible for the stability of the crystal lattice. ${ }^{44-47}$ The bigger the number of hydrogen bonds, the greater the reticular stability. ${ }^{48}$ Intuitively, it is possible to think that the robustness of the materials could be negatively influenced by the introduction of an ammonium salt derived from a secondary amine as a consequence of the decrease of the number of hydrogen bonds, three in conventional ammonium salts versus two in this class of cations, see Scheme 1. Indeed, it could be reasonable to expect the total degradation of the material as an outcome of the decrease in the number of hydrogen bonds. On this concern, we focus on the determination of the stability through a thermal stability study of the films with $\mathrm{n}=10,50$ and 90, using 3D perovskite film, as reference. Bearing in mind the proposed mechanisms of degradation of hybrid organic-inorganic lead iodide perovskites, ${ }^{49,50}$ and considering that the sub-products are ammonia/amines together with $\mathrm{PbI}_{2},{ }^{51}$ we determine to use infrared (IR) spectroscopy to follow the perovskite degradation through the diffusion of ammonia/amines to atmosphere. Considering the vanishing of any absorption peak as an indicator of material degradation and the simplicity and 
velocity with which an IR spectrum can be acquired, we carried out a thermal stability study monitoring the fading of the strong peaks above $3000 \mathrm{~cm}^{-1}$, shaded in Figure $4 \mathrm{a}$, in the IR spectra. The films were annealed in air with an ambient humidity between 50$60 \%$ at $\mathrm{T}=100{ }^{\circ} \mathrm{C}$ for three periods of 80 minutes each of them, acquiring the corresponding IR spectra at the end of each period. The IR spectrum for 3D perovskite exhibits the characteristics N-H stretching absorption peaks for primary amines at 3176 $\mathrm{cm}^{-1}$ and $3131 \mathrm{~cm}^{-1}$, and in-plane $\mathrm{NH}_{2}$ scissoring absorptions at $1467 \mathrm{~cm}^{-1}$. Despite the slight difference in chemical composition between the examined materials, the IR spectra essentially exhibit identical features at the initial time $(t=0)$ for all the analyzed samples, see Figure 4a. Although the coloration of the films remains apparently unaffected, at least after $80 \mathrm{~min}$ of heating time, it was possible to detect that the peak above $3000 \mathrm{~cm}^{-1}$ decreases monotonically as the heating time progresses, see Figure $4 \mathrm{~d}$. Finally, after a heating time of $240 \mathrm{~min}$, the relative concentration of perovskite for the reference $3 \mathrm{D}$ sample is just $\sim 8 \%$ of the initial concentration of perovskite material.

(a)

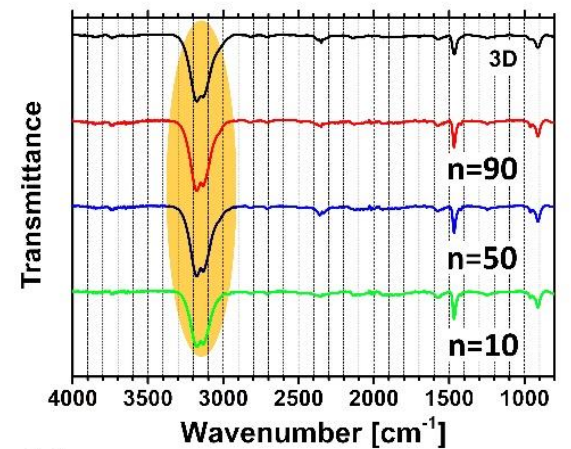

(c)

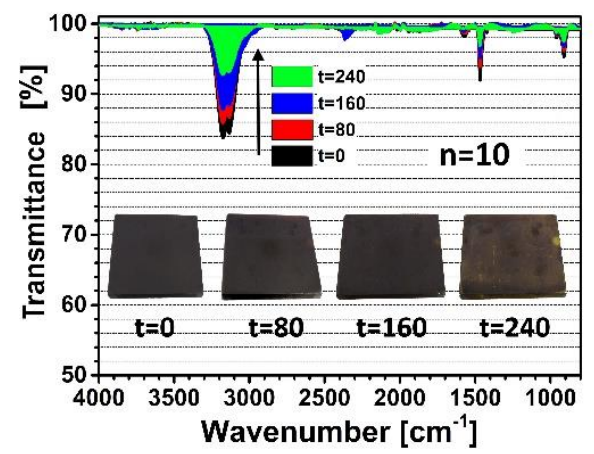

(b)

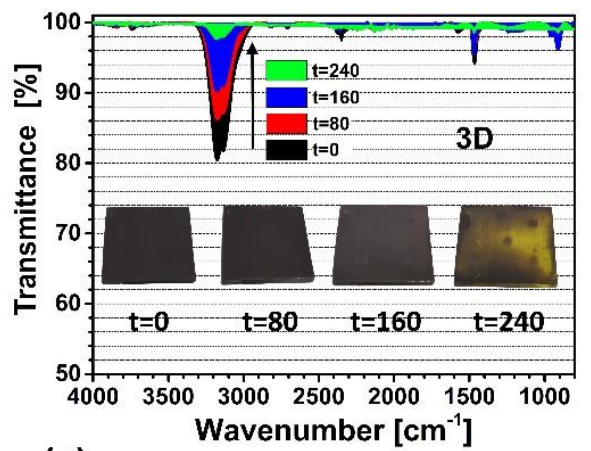

(d)

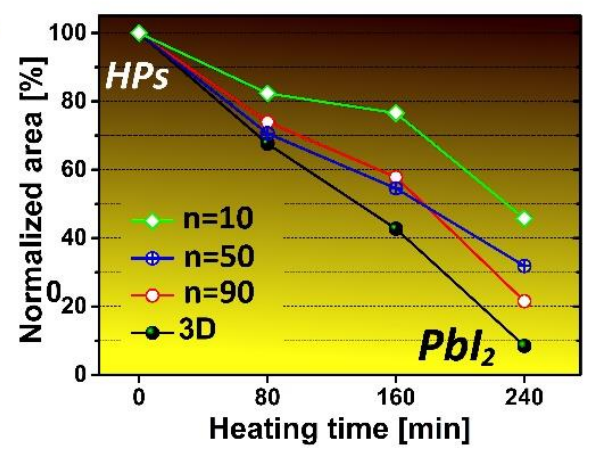

Figure 4. Thermal stability evaluated by IR spectroscopy $\mathrm{T}=100^{\circ} \mathrm{C} ; \mathrm{t}=80 \mathrm{~min}$ ( 3 times) in air with an ambient humidity ranging from 50 to $60 \%$. (a) IR spectra for the evaluated samples. The highlighted fragment points out the resonances owing to $\mathrm{N}-\mathrm{H}$ stretching, which we study to follow the loss of ammonium salts. (b) and (c) Evolution of $\mathrm{N}-\mathrm{H}$ peak on ammonium salts as function of heating time; $t$ is in minutes. (d) Qualitative determination of the concentration of ammonium salts (and therefore perovskite material) by the integration of the N-H peak. 
Considering that $2 \mathrm{D} / 3 \mathrm{D}$ HPs with $\mathrm{n}=90$ is a material whose properties are very near to the 3D limit, the behavior should be very similar to 3D HPs. Interestingly, it was possible to detect a higher thermal resistant property than 3D HPs through the entire range of studied time, see Figure 4b, d, and S5. After 240 min of heating exposition, $n=90$ sample presented near $22 \%$ of relative concentration of 2D/3D HPs, almost $275 \%$ comparing with 3D HP, which demonstrate the dramatic effect induced by the presence of dipropylammonium iodide. Importantly, this stabilization effect is not observed with the introduction of formamidinium (FA). ${ }^{52}$ This comparison is especially important considering the lower tendency of FA to release hydrogen ions than MA, avoiding the formation of $\mathrm{HI}$ and therefore the degradation of the material. A related tendency was detected for $\mathrm{n}=50$ in the medium range (from 0-160 min). However, that sample shows a concentration four times greater than 3D HP after $t=240 \mathrm{~min}$, see Figure S6. In agreement with these results, for the material with $n=10$, the higher concentration of dipropylammonium iodide leads to obtain a remarkable effect. While the other materials lose almost $30 \%$ and more of $40 \%$ of ammonium salts at $\mathrm{t}=80 \mathrm{~min}$ and $160 \mathrm{~min}$, respectively, $n=10$ almost keeps $80 \%$ of its original concentration, see Figure $4 \mathrm{~b}, \mathrm{c}$ and d. After $240 \mathrm{~min}, \mathrm{n}=10$ retains near $50 \%$ of the bare concentration, which means an increment of $575 \%$ comparing with 3D HP, see Figure 4c, d and Table 3. More stressing conditions were also evaluated, exposing the films to $120^{\circ} \mathrm{C}$ for two periods of $80 \mathrm{~min}$, see Figure S7. In this case, after $80 \mathrm{~min}$ of annealing, the materials with dipropylammonium cation ( $\mathrm{n}=10,50$ and 90$)$ exhibit near $80 \%$ of the primary material. In contrast, 3D HPs based films show only 50\%. When the annealing was extended by an additional period of $80 \mathrm{~min}$, it was possible to detect that 3D HPs degraded totally ( $0 \%$ detection of amine), while for 2D/3D HPs films with $n=50$ and 90 , it could detect until a $20 \%$ of the perovskite material. Finally, for the material with $n=10$, the IR spectra display a slightly upper to $30 \%$ of the perovskite phase, see Figure S7.

Table 3. Relative concentration of methylammonium cation extracted from the integration of the bands above $3000 \mathrm{~cm}^{-1}$ in the IR spectra.

\begin{tabular}{|c|c|c|c|c|}
\hline $\mathbf{T}$ & 3D HP & $\mathbf{n = 9 0}$ & $\mathbf{n = 5 0}$ & $\mathbf{n = 1 0}$ \\
$(\mathbf{m i n})$ & $(\%)$ & $(\%)$ & $(\%)$ & $(\%)$ \\
\hline
\end{tabular}




\begin{tabular}{|c|c|c|c|c|}
\hline 0 & 100 & 100 & 100 & 100 \\
\hline 80 & 68 & 74 & 71 & 82 \\
\hline 160 & 43 & 58 & 55 & 77 \\
\hline 240 & 8 & 22 & 32 & 46 \\
\hline
\end{tabular}

With the results outlined so far, we demonstrate that the use of dipropylammonium, a smaller cation than those used conventionally, as butylammonium, hexylammonium, etc. could lead to obtain materials that, additionally to preserve properties as hydrophobicity, present also long-carrier's lifetimes. Justly, this last observation strongly supports its successful application as light-harvester in solar cells.

In order to evaluate further, not just the stability of the material but the stability of the device, PSCs based on the different materials were also subjected to post-thermal treatment to evaluate the relationship between the thermal stability and device performance. PSCs performance was evaluated for fresh samples and after an annealing of the whole device at $60{ }^{\circ} \mathrm{C}$ for 30 minutes in air with $45 \%$ humidity with no encapsulation. PSC efficiency was measured by the determination of the PSC stabilized efficiency after $1000 \mathrm{~s}$ at continuous 1 sun illumination at maximum power point, see Figure S8 and Table 4. It is remarkable that the efficiencies of the perovskite with $n=10$ but also with a high $\mathrm{n}$ value as $\mathrm{n}=50$ are maintained, with an efficiency drop lower than 4\%, after the stressing annealing. The stabilized device efficiency, see Table 4, shows how the presence of the dipropylammonium increases the thermal stability, in accordance with the results shown on the bare material. In fact, even when amount of dipropylammonium is relatively small for $n=90$, the efficiency drop after thermal annealing decreases from $83 \%$ for $3 \mathrm{D}$ reference samples to $41 \%$ for $n=90$. Nevertheless, the increase of device stabilization by the dipropylammonium is dramatic in the case of $\mathrm{n}=50$ and $\mathrm{n}=10$, with an efficiency drop of just $3.6 \%$ and $3.4 \%$ respectively, see Table 4. Although the stability tests on the devices involve a lot of variables and mechanisms, beyond the study reported here, these results highlight that the material based on the mixture of dipropylammonium and methylammoniun cations released the thermal stress, and above all confirms the application utility of a secondary ammine in the thermal stabilization of not just de material but the device. 
Moreover, PCE obtained from the J-V curve reverse and forward scans of the devices based on $\operatorname{Dip}_{2} \mathrm{MA}_{\mathrm{n}-1} \mathrm{~Pb}_{\mathrm{n}} \mathrm{I}_{3 \mathrm{n}+1}$ with $\mathrm{n}=10,50$ and 90 and 3D are also shown in Figure S8 and Table S1, with the corresponding PCE measured from stabilized maximum power point tracking after 1000s. Note that the reverse scan overestimates the PCE less than $8 \%$ for non-severely degraded devices, and just for degraded 3D device after thermal analysis there is a significant discrepancy between the PCE calculated reverse J-V scan and from stabilized measurement, see Table S1.

Table 4. Photoconversion efficiencies tracked for 1000 seconds at the maximum power point, and the efficiencies drop of the devices based on $\operatorname{Dip}_{2} \mathrm{MA}_{n-1} \mathrm{~Pb}_{n} \mathrm{I}_{3 n+1}$ with $\mathrm{n}=10$, 50 and 90 and 3D, before and after the thermal treatment at $60{ }^{\circ} \mathrm{C}$ for 30 minutes in air, $45 \%$ humidity, devices were not encapsulated.

\begin{tabular}{|c|c|c|c|c|}
\hline & 3D HP & $\mathbf{n = 9 0}$ & $\mathbf{n = 5 0}$ & $\mathbf{n = 1 0}$ \\
\hline PCE $_{\text {MPP }} \mathbf{( \% )}$ & 15.08 & 14.50 & 13.86 & 12.53 \\
\hline PCE $_{\text {MPP }}\left(\mathbf{\% )} \mathbf{T}^{\mathbf{T}} \mathbf{6 0}{ }^{\circ} \mathrm{C}\right.$ & 2.61 & 8.59 & 13.36 & 12.10 \\
\hline Efficiency drop (\%) $^{\mid}$ & 82.7 & 40.7 & 3.6 & 3.4 \\
\hline
\end{tabular}

To explain the observed behavior, our hypothesis was centered on the higher number of carbon chains in the dipropylammonium cation than methylammonium salt. The results obtained by thermogravimetric analysis (TGA) demonstrate that, comparing salts, dipropylammonium iodide sublimates slightly before methylammonium iodide, see Figure S9. Nevertheless, when a similar analysis was carried out for the material with $n=90$, we could determine a slightly higher sublimation's temperature for 2D/3D HPs with $n=90$ than the observed in 3D perovskites, see Figure S10. This fact points out that the advantageous influence of dipropylammonium is just detectable in the solid state, after the packing of 2D/3D perovskite was carried out. To examine the influence that the small size as well as the closeness and therefore the potential interactions of the chains among each other when it was used dipropylammonium iodide as bulky cation, we carried out theoretical simulations based on density functional theory. Considering that our goal is the determination of interactions between the bulky cations with themselves and with the inorganic slides, we decided to study just the $2 \mathrm{D}$ HPs with $n=1$. Furthermore, the computational requirements are considerably lower than the necessary for superior 2D/3D HPs. We observed shorter distances between the organic chains on both, itself and with neighboring molecules, than those observed in 2D HPs fabricated with solely primary ammonium salts, as butylammonium, ${ }^{53}$ see Figure 5, Figure S11 and Table S2. Figure S11 compare the crystalline structure for dipropylammonium 
cation and butylammonium cation for $n=1$, different views are provided for comparison and for highlight the layered 2D structure of both cations.

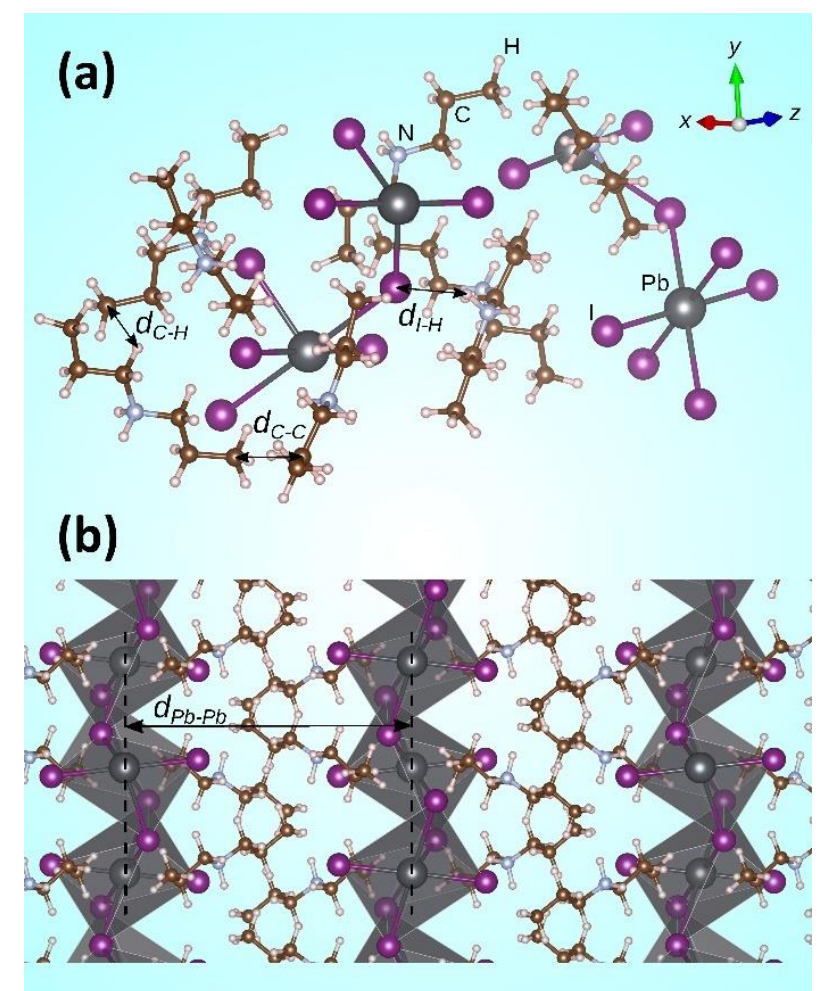

Figure 5. Atomic representation of a $2 D$ HPs with $n=1$ using dipropylammonium cation. Panel (a), calculated unit cell. In panel (b), bulk structure with tilted $\mathrm{PbI}_{6}$ octahedra. We indicate the relevant distances as reported in Table S1.

Importantly, we could further determine that the interlayer distance between neighboring inorganic slides in the dipropylammnonium based material is $12.83 \AA$, slightly shorter than $13.11 \AA$, which is the interplanar distance determined experimentally for the 2D HPs with butylammonium as bulky cation. The determined lattice parameters for dipropylammonium iodide are $a=9.37 \AA$, $b=10.20 \AA$, and $c=25.64$ $\AA$. In contrast, the unit cell for butylammonium, experimentally determined, shows values of $a=8.43 \AA, b=8.99 \AA$, and $c=26.23 \AA .{ }^{53}$ From these data, we can deduce that the distribution of chains in two arms instead of just one, could elongate the $\mathrm{x}$ and $\mathrm{y}$ axes, see Figure 5a for the axis reference. However, the $\mathrm{z}$ axe, which eventually determines the electronic coupling or disruption and therefore the electronic transport, is abridged. Consequently, we hypothesize that the observed optical properties (absence of excitons at room temperature), as well as the high performance observed in our 
material, can be related a reduction in the quantum and dielectric confinement effects. Concerning the stability, these results suggest that the unexpected high stability attained in 2D/3D HPs prepared with dipropylammonium could be related to the larger number of interactions (mainly van der Waals) reducing the predicted negative impact owing to the lower number of hydrogen bonds. However, further crystallographic studies are desirable to determine the crystal packing pattern that seems to be responsible for the improvement of the stability.

In summary, we report the preparation, study, and application of a new $\operatorname{Dip}_{2} \mathrm{MA}_{\mathrm{n}-1} \mathrm{~Pb}_{\mathrm{n}} \mathrm{I}_{3 \mathrm{n}+1}$ 2D/3D perovskite family in the fabrication of thermally stable and highly efficient solar cells based on secondary amine bulky cations. Through optical results, we demonstrate that the size of the cation modifies the charge carrier's dynamics and it was possible to establish that the application of bulky cations with a small size will lead to obtain materials with improved capacities as long carrier lifetimes and therefore higher PCE. More importantly, we demonstrate that the inclusion of an ammonium salt derived from a secondary amine could be not only adequate but also desirable to obtain materials and devices with significantly higher thermal stability than 3D perovskites. The demonstration of the suitability of secondary ammonium salt for the preparation of high-performance perovskites significantly widens the range of 2D/3D perovskite materials for optoelectronic applications. Besides, the feasibility of include two organic groups instead of only one on the ammonium head, with similar or different properties, will rich the scope of opportunities to the optimization of the materials and optoelectronic devices by a highly specific fine-tuning and functionalization.

Acknowledgment. This work was supported by the University Jaume I (project SOLENPE UJI-B2016-05), the Ministerio de Economia y Competividad of Spain Government (MAT2016-76892-C3-1-R) and European Research Council (ERC) via Consolidator Grant (724424 - No-LIMIT). J.A.S.D. is thankful with SENERCONACyT by financial support

\section{Supporting Information}

Materials and methods, NMR of the prepared ammonium salt, TCSPC measurements with the corresponding fittings, MPP tracking, determination of concentration of 
ammonium salts for the materials with $\mathrm{n}=50,90$ by IR, TGA results, atomic representation of 2D HPs using dipropil and butylammonium iodide and the table with the comparison of interatomic distances of DipI and butyl based HPs can be found in the supporting information.

\section{References}

(1) Fu, Q.; Tang, X.; Huang, B.; Hu, T.; Tan, L.; Chen, L.; Chen, Y.: Recent Progress on the Long-Term Stability of Perovskite Solar Cells. Advanced Science, 1700387-n/a.

(2) Leijtens, T.; Eperon, G. E.; Noel, N. K.; Habisreutinger, S. N.; Petrozza, A.; Snaith, H. J.: Stability of Metal Halide Perovskite Solar Cells. Advanced Energy Materials 2015, 5, 1500963.

(3) Luo, S.; Daoud, W. A.: Recent progress in organic-inorganic halide perovskite solar cells: mechanisms and material design. Journal of Materials Chemistry A 2015, 3, 89929010 .

(4) Aristidou, N.; Sanchez-Molina, I.; Chotchuangchutchaval, T.; Brown, M.; Martinez, L.; Rath, T.; Haque, S. A.: The Role of Oxygen in the Degradation of Methylammonium Lead Trihalide Perovskite Photoactive Layers. Angewandte Chemie International Edition 2015, 54, 8208-8212.

(5) Smith, I. C.; Hoke, E. T.; Solis-Ibarra, D.; McGehee, M. D.; Karunadasa, H. I.: A Layered Hybrid Perovskite Solar-Cell Absorber with Enhanced Moisture Stability. Angewandte Chemie International Edition 2014, 53, 11232-11235.

(6) Quan, L. N.; Yuan, M.; Comin, R.; Voznyy, O.; Beauregard, E. M.; Hoogland, S.; Buin, A.; Kirmani, A. R.; Zhao, K.; Amassian, A.; Kim, D. H.; Sargent, E. H.: LigandStabilized Reduced-Dimensionality Perovskites. Journal of the American Chemical Society 2016, 138, 2649-55.

(7) Cao, D. H.; Stoumpos, C. C.; Farha, O. K.; Hupp, J. T.; Kanatzidis, M. G.: 2D Homologous Perovskites as Light-Absorbing Materials for Solar Cell Applications. Journal of the American Chemical Society 2015, 137, 7843-50.

(8) Mao, L.; Ke, W.; Pedesseau, L.; Wu, Y.; Katan, C.; Even, J.; Wasielewski, M. R.; Stoumpos, C. C.; Kanatzidis, M. G.: Hybrid Dion-Jacobson 2D Lead Iodide Perovskites. Journal of the American Chemical Society 2018, 140, 3775-3783.

(9) Soe, C. M. M.; Stoumpos, C. C.; Kepenekian, M.; Traore, B.; Tsai, H.; Nie, W.; Wang, B.; Katan, C.; Seshadri, R.; Mohite, A. D.; Even, J.; Marks, T. J.; Kanatzidis, M. G.: New Type of 2D Perovskites with Alternating Cations in the Interlayer Space, $(\mathrm{C}(\mathrm{NH} 2) 3)(\mathrm{CH} 3 \mathrm{NH} 3) n \mathrm{PbnI} 3 \mathrm{n}+1$ : Structure, Properties, and Photovoltaic Performance. Journal of the American Chemical Society 2017, 139, 16297-16309.

(10) Mitzi, D. B., Chondroudis, K., Kagan, C.R.: Organic-inorganic electronics. IBM $J$ Res \& Dev 2001, 29-45.

(11) Grancini, G.; Roldan-Carmona, C.; Zimmermann, I.; Mosconi, E.; Lee, X.; Martineau, D.; Narbey, S.; Oswald, F.; De Angelis, F.; Graetzel, M.; Nazeeruddin, M. K.: OneYear stable perovskite solar cells by 2D/3D interface engineering. Nature communications 2017, 8, 15684 .

(12) Tsai, H.; Nie, W.; Blancon, J.-C.; Stoumpos, C. C.; Asadpour, R.; Harutyunyan, B.; Neukirch, A. J.; Verduzco, R.; Crochet, J. J.; Tretiak, S.; Pedesseau, L.; Even, J.; Alam, M. A.; Gupta, G.; Lou, J.; Ajayan, P. M.; Bedzyk, M. J.; Kanatzidis, M. G.; Mohite, A. D.: Highefficiency two-dimensional Ruddlesden-Popper perovskite solar cells. Nature 2016, 536, 312.

(13) Yang, F.; Zhang, P.; Kamarudin, M. A.; Kapil, G.; Ma, T.; Hayase, S.: Addition Effect of Pyreneammonium Iodide to Methylammonium Lead Halide Perovskite-2D/3D Heterostructured Perovskite with Enhanced Stability. Advanced Functional Materials 2018, 28, 1804856. 
(14) Zhou, J.; Chu, Y.; Huang, J.: Photodetectors Based on Two-Dimensional LayerStructured Hybrid Lead Iodide Perovskite Semiconductors. ACS Applied Materials \& Interfaces 2016, $8,25660-25666$.

(15) Zhang, X.; Wang, C.; Zhang, Y.; Zhang, X.; Wang, S.; Lu, M.; Cui, H.; Kershaw, S. V.; Yu, W. W.; Rogach, A. L.: Bright Orange Electroluminescence from Lead-Free Two-Dimensional Perovskites. ACS Energy Letters 2019, 4, 242-248.

(16) Cheng, P.; Xu, Z.; Li, J.; Liu, Y.; Fan, Y.; Yu, L.; Smilgies, D.-M.; Müller, C.; Zhao, K.; Liu, S. F.: Highly Efficient Ruddlesden-Popper Halide Perovskite PA2MA4Pb5I16 Solar Cells. ACS Energy Letters 2018, 3, 1975-1982.

(17) Proppe, A. H.; Quintero-Bermudez, R.; Tan, H.; Voznyy, O.; Kelley, S. O.; Sargent, E. H.: Synthetic Control over Quantum Well Width Distribution and Carrier Migration in Low-Dimensional Perovskite Photovoltaics. Journal of the American Chemical Society 2018, 140, 2890-2896.

(18) Yang, F.; Zhang, P.; Kamarudin, M. A.; Kapil, G.; Ma, T.; Hayase, S.: Addition Effect of Pyreneammonium Iodide to Methylammonium Lead Halide Perovskite-2D/3D Heterostructured Perovskite with Enhanced Stability. Advanced Functional Materials 2018, 0, 1804856.

(19) Gao, Y.; Shi, E.; Deng, S.; Shiring, S. B.; Snaider, J. M.; Liang, C.; Yuan, B.; Song, R.; Janke, S. M.; Liebman-Peláez, A.; Yoo, P.; Zeller, M.; Boudouris, B. W.; Liao, P.; Zhu, C.; Blum, V.; Yu, Y.; Savoie, B. M.; Huang, L.; Dou, L.: Molecular engineering of organic-inorganic hybrid perovskites quantum wells. Nature Chemistry 2019, 11, 1151-1157.

(20) Rodríguez-Romero, J.; Hames, B. C.; Mora-Seró, I.; Barea, E. M.: Conjugated Organic Cations to Improve the Optoelectronic Properties of 2D/3D Perovskites. ACS Energy Letters 2017, 2, 1969-1970.

(21) Rodríguez-Romero, J.; Clasen Hames, B.; Galar, P.; Fakharuddin, A.; Suarez, I.; Schmidt-Mende, L.; Martínez-Pastor, J. P.; Douhal, A.; Mora-Seró, I.; Barea, E. M.: Tuning optical/electrical properties of 2D/3D perovskite by the inclusion of aromatic cation. Physical Chemistry Chemical Physics 2018, 20, 30189-30199.

(22) Mitzi, D. B.; Chondroudis, K.; Kagan, C. R.: Design, Structure, and Optical Properties of Organic-Inorganic Perovskites Containing an Oligothiophene Chromophore. Inorganic Chemistry 1999, 38, 6246-6256.

(23) Van Gompel, W. T. M.; Herckens, R.; Van Hecke, K.; Ruttens, B.; D'Haen, J.; Lutsen, L.; Vanderzande, D.: Towards 2D layered hybrid perovskites with enhanced functionality: introducing charge-transfer complexes via self-assembly. Chemical Communications 2019, 55, 2481-2484.

(24) Ortiz-Cervantes, C.; Román-Román, P. I.; Vazquez-Chavez, J.; HernándezRodríguez, M.; Solis-Ibarra, D.: Thousand-fold Conductivity Increase in 2D Perovskites by Polydiacetylene Incorporation and Doping. Angewandte Chemie 2018, 130, 14078-14082.

(25) Ortiz-Cervantes, C.; Carmona-Monroy, P.; Solis-Ibarra, D.: Two-Dimensional Halide Perovskites in Solar Cells: 2D or not 2D? ChemSusChem 2019, 12, 1560-1575.

(26) Xu, Z.; Mitzi, D. B.; Medeiros, D. R.: [(CH3)3NCH2CH2NH3]SnI4: A Layered Perovskite with Quaternary/Primary Ammonium Dications and Short Interlayer Iodine-Iodine Contacts. Inorganic Chemistry 2003, 42, 1400-1402.

(27) Jung, M.-H.: Hydrophobic perovskites based on an alkylamine compound for high efficiency solar cells with improved environmental stability. Journal of Materials Chemistry A 2019, 7, 14689-14704.

(28) Vega, E.; Mollar, M.; Marí, B.: Effect of guanidinium on the optical properties and structure of the methylammonium lead halide perovskite. Journal of Alloys and Compounds 2018, 739, 1059-1064.

(29) Blancon, J. C.; Tsai, H.; Nie, W.; Stoumpos, C. C.; Pedesseau, L.; Katan, C.; Kepenekian, M.; Soe, C. M.; Appavoo, K.; Sfeir, M. Y.; Tretiak, S.; Ajayan, P. M.; Kanatzidis, M. G.; Even, J.; Crochet, J. J.; Mohite, A. D.: Extremely efficient internal exciton dissociation through edge states in layered 2D perovskites. Science 2017, 355, 1288-1292. 
(30) Cohen, B.-E.; Wierzbowska, M.; Etgar, L.: High Efficiency and High Open Circuit Voltage in Quasi 2D Perovskite Based Solar Cells. Advanced Functional Materials 2017, 27, 1604733-n/a.

(31) De Marco, N.; Zhou, H.; Chen, Q.; Sun, P.; Liu, Z.; Meng, L.; Yao, E.-P.; Liu, Y.; Schiffer, A.; Yang, Y.: Guanidinium: A Route to Enhanced Carrier Lifetime and OpenCircuit Voltage in Hybrid Perovskite Solar Cells. Nano Letters 2016, 16, 1009-1016.

(32) Mao, L.; Tsai, H.; Nie, W.; Ma, L.; Im, J.; Stoumpos, C. C.; Malliakas, C. D.; Hao, F.; Wasielewski, M. R.; Mohite, A. D.; Kanatzidis, M. G.: Role of Organic Counterion in Lead- and Tin-Based Two-Dimensional Semiconducting Iodide Perovskites and Application in Planar Solar Cells. Chemistry of Materials 2016, 28, 7781-7792.

(33) Zhang, F.; Kim, D. H.; Lu, H.; Park, J.-S.; Larson, B. W.; Hu, J.; Gao, L.; Xiao, C.; Reid, O. G.; Chen, X.; Zhao, Q.; Ndione, P. F.; Berry, J. J.; You, W.; Walsh, A.; Beard, M. C.; Zhu, K.: Enhanced Charge Transport in 2D Perovskites via Fluorination of Organic Cation. Journal of the American Chemical Society 2019, 141, 5972-5979.

(34) Soe, C. M. M.; Stoumpos, C. C.; Kepenekian, M.; Traoré, B.; Tsai, H.; Nie, W.; Wang, B.; Katan, C.; Seshadri, R.; Mohite, A. D.; Even, J.; Marks, T. J.; Kanatzidis, M. G.: New Type of 2D Perovskites with Alternating Cations in the Interlayer Space, $(\mathrm{C}(\mathrm{NH} 2) 3)(\mathrm{CH} 3 \mathrm{NH} 3) \mathrm{nPbnI} \mathrm{n}+1$ : Structure, Properties, and Photovoltaic Performance. Journal of the American Chemical Society 2017, 139, 16297-16309.

(35) Chen, Q.; Zhou, H.; Song, T.-B.; Luo, S.; Hong, Z.; Duan, H.-S.; Dou, L.; Liu, Y.; Yang, Y.: Controllable Self-Induced Passivation of Hybrid Lead Iodide Perovskites toward High Performance Solar Cells. Nano Letters 2014, 14, 4158-4163.

(36) Ishihara, T.; Takahashi, J.; Goto, T.: Optical properties due to electronic transitions in two-dimensional semiconductors $\left(\$\{\backslash \operatorname{mathrm}\{\mathrm{C}\}\}_{-}\{\backslash\right.$ mathit $\{\mathrm{n}\}\} \$ \$\{\text { mathrm }\{\mathrm{H}\}\}_{-}\{2 \backslash \operatorname{mathit}\{\mathrm{n}\}+1\} \$ \$\{\backslash \operatorname{mathrm}\{\mathrm{NH}\}\}_{-}\{3\} \$ \$\{)$ \}$_{-}\{2\} \$ \$\{\text { Imathrm }\{\mathrm{PbI}\}\}_{-}\{4\} \$$. Physical Review B 1990, 42, 11099-11107.

(37) Zheng, K.; Chen, Y.; Sun, Y.; Chen, J.; Chábera, P.; Schaller, R.; Al-Marri, M. J.; Canton, S. E.; Liang, Z.; Pullerits, T.: Inter-phase charge and energy transfer in RuddlesdenPopper 2D perovskites: critical role of the spacing cations. Journal of Materials Chemistry A 2018, 6, 6244-6250.

(38) Stranks, S. D.; Burlakov, V. M.; Leijtens, T.; Ball, J. M.; Goriely, A.; Snaith, H. J.: Recombination Kinetics in Organic-Inorganic Perovskites: Excitons, Free Charge, and Subgap States. Physical Review Applied 2014, 2, 034007.

(39) Méndez, P. F.; Muhammed, S. K. M.; Barea, E. M.; Masi, S.; Mora-Seró, I.: Analysis of the UV-Ozone-Treated SnO2 Electron Transporting Layer in Planar Perovskite Solar Cells for High Performance and Reduced Hysteresis. Solar RRL 2019, 3, 1900191.

(40) Ishihara, T.; Takahashi, J.; Goto, T.: Exciton state in two-dimensional perovskite semiconductor (C10H21NH3)2PbI4. Solid State Communications 1989, 69, 933-936.

(41) Hong, X.; Ishihara, T.; Nurmikko, A. V.: Dielectric confinement effect on excitons in $\$\{\backslash \text { mathrm }\{\mathrm{PbI}\}\}_{-}\{4\}$ \$-based layered semiconductors. Physical Review B 1992, 45, 6961-6964.

(42) Hirasawa, M.; Ishihara, T.; Goto, T.: Exciton Features in 0-, 2-, and 3Dimensional Networks of [PbI6]4- Octahedra. Journal of the Physical Society of Japan 1994, 63, 3870-3879.

(43) Tsai, H.; Asadpour, R.; Blancon, J.-C.; Stoumpos, C. C.; Even, J.; Ajayan, P. M.; Kanatzidis, M. G.; Alam, M. A.; Mohite, A. D.; Nie, W.: Design principles for electronic charge transport in solution-processed vertically stacked 2D perovskite quantum wells. Nature Communications 2018, 9, 2130.

(44) Sourisseau, S.; Louvain, N.; Bi, W.; Mercier, N.; Rondeau, D.; Boucher, F.; Buzaré, J.-Y.; Legein, C.: Reduced Band Gap Hybrid Perovskites Resulting from Combined Hydrogen and Halogen Bonding at the Organic-Inorganic Interface. Chemistry of Materials 2007, 19, 600-607.

(45) Mercier, N.; Poiroux, S.; Riou, A.; Batail, P.: Unique Hydrogen Bonding Correlating with a Reduced Band Gap and Phase Transition in the Hybrid Perovskites $(\mathrm{HO}(\mathrm{CH} 2) 2 \mathrm{NH} 3) 2 \mathrm{PbX} 4(\mathrm{X}=\mathrm{I}, \mathrm{Br})$. Inorganic Chemistry 2004, 43, 8361-8366. 
(46) Ono, L. K.; Park, N.-G.; Zhu, K.; Huang, W.; Qi, Y.: Perovskite Solar CellsTowards Commercialization. ACS Energy Letters 2017, 2, 1749-1751.

(47) Mercier, N.: (HO2C(CH2)3NH3)2(CH3NH3)Pb2I7: a predicted noncentrosymmetrical structure built up from carboxylic acid supramolecular synthons and bilayer perovskite sheets. CrystEngComm 2005, 7, 429-432.

(48) Mitzi, D. B.; Liang, K.: Synthesis, Resistivity, and Thermal Properties of the Cubic Perovskite NH2CH=NH2SnI3and Related Systems. Journal of Solid State Chemistry 1997, 134, 376-381.

(49) Juarez-Perez, E. J.; Hawash, Z.; Raga, S. R.; Ono, L. K.; Qi, Y.: Thermal degradation of $\mathrm{CH} 3 \mathrm{NH} 3 \mathrm{PbI} 3$ perovskite into $\mathrm{NH} 3$ and $\mathrm{CH} 3 \mathrm{I}$ gases observed by coupled thermogravimetry-mass spectrometry analysis. Energy \& Environmental Science 2016, 9, 3406-3410.

(50) Williams, A. E.; Holliman, P. J.; Carnie, M. J.; Davies, M. L.; Worsley, D. A.; Watson, T. M.: Perovskite processing for photovoltaics: a spectro-thermal evaluation. Journal of Materials Chemistry A 2014, 2, 19338-19346.

(51) Ciccioli, A.; Latini, A.: Thermodynamics and the Intrinsic Stability of Lead Halide Perovskites CH3NH3PbX3. The Journal of Physical Chemistry Letters 2018, 9, 37563765 .

(52) Tan, W.; Bowring, A. R.; Meng, A. C.; McGehee, M. D.; McIntyre, P. C.: Thermal Stability of Mixed Cation Metal Halide Perovskites in Air. ACS Applied Materials \& Interfaces 2018, 10, 5485-5491.

(53) Billing, D. G.; Lemmerer, A.: Synthesis, characterization and phase transitions in the inorganic-organic layered perovskite-type hybrids [(CnH2n $+1 \mathrm{NH} 3) 2 \mathrm{PbI} 4], \mathrm{n}=4,5$ and 6. Acta Crystallographica Section B 2007, 63, 735-747.

\section{TOC Figure}

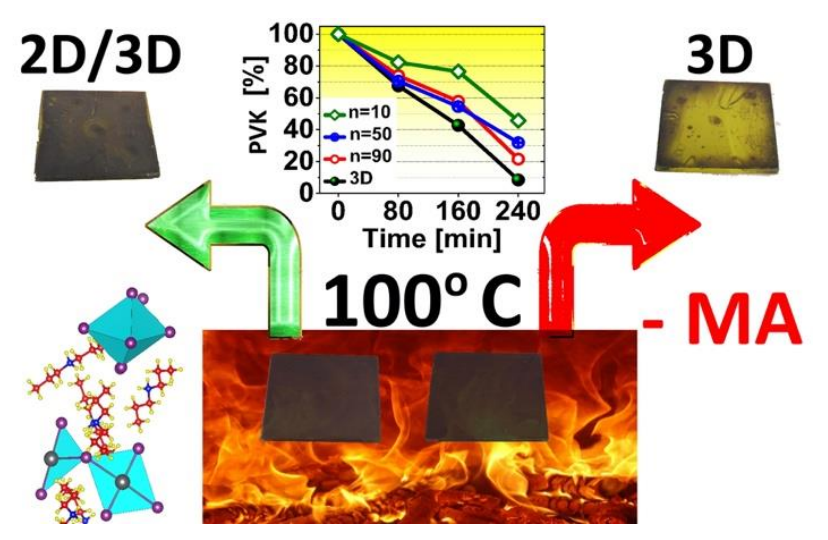

